\title{
Atividade residual de herbicidas pré-emergentes aplicados em solos contrastantes $^{1}$
}

\author{
Residual activity of pre-emergent herbicides applied in contrasting soils
}

\author{
Miriam Hiroko Inoue ${ }^{2}$, Kassio Ferreira Mendes ${ }^{3}$, Cleverton Tiago Carneiro de Santana ${ }^{4}$, Ana \\ Cássia Silva Possamai ${ }^{5}$
}

Resumo - O trabalho objetivou estudar a atividade residual de diferentes doses dos herbicidas alachlor, oxyfluorfen, prometryne e S-metolachlor em solos com texturas contrastantes, aplicadas em pré-emergência. Para tanto oito ensaios simultâneos foram realizados, com amostras de NEOSSOLO QUARTZARÊNICO (textura arenosa) e LATOSSOLO VERMELHO (textura argilosa), que receberam aplicações de alachlor $\left(0 ; 2,40 ;\right.$ e $\left.3,36 \mathrm{~kg} \mathrm{ha}^{-1}\right)$, oxyfluorfen $(0 ; 0,48$; e $\left.0,72 \mathrm{~kg} \mathrm{ha}^{-1}\right)$, prometryne $\left(0 ; 0,75\right.$; e $\left.1,50 \mathrm{~kg} \mathrm{ha}^{-1}\right)$ e S-metolachlor $\left(0 ; 1,20\right.$; e 1,44 $\left.\mathrm{kg} \mathrm{ha}^{-1}\right)$. O monitoramento dos tratamentos foi a partir da semeadura de um bioindicador (Brachiaria decumbens ou Cucumis sativus) aos 0, 25, 50, 75 e 100 dias após a aplicação (DAA) dos herbicidas, avaliando o controle aos 45 dias após a semeadura por meio de notas de 0 à $100 \%$. Independente da dose, o alachlor proporcionou controle acima de $80 \%$ até 66 DAA, em solo argiloso, e até 50 DAA, em arenoso. Oxyfluorfen evidenciou controle acima de $80 \%$ até 56 DAA para solo argiloso e até 36 DAA para arenoso, em ambas as doses. Ambas as doses de prometryne em solo argiloso proporcionoram controle acima de $80 \%$ até 28 DAA, com maior atividade residual na dose recomendada $\left(1,50 \mathrm{~kg} \mathrm{ha}^{-1}\right)$. Nas amostras arenosas, S-metolachlor proporcionou o mesmo controle para as duas doses, controlando o bioindicador em $80 \%$ até 52 DAA, e para o solo argiloso na dose $1,44 \mathrm{~kg} \mathrm{ha}^{-1}$, apresentou $96 \%$ de controle até 80 DAA. Desta forma, em solo arenoso não houve aumento da atividade residual, mesmo com a aplicação da dose recomendada para solo argiloso.

Palavras-Chave: Bioindicadores, persistência, textura do solo.

Abstract - This study investigated the residual activity of different rates of the herbicides alachlor, oxyfluorfen, prometryne and S-metolachlor applied in pre-emergence in soils of contrasting textures. For that, eight simultaneous experiments were conducted with samples of PSAMENT (sandy texture) and RED LATOSOL (clay texture), which received alachlor $(0 ; 2.40$

\footnotetext{
${ }^{1}$ Recebido para publicação em 10/08/2011 e aceito em 12/12/2011.

2 Eng $^{\circ}$ Agr $^{\circ}$, D.Sc., Professora do Departamento de Agronomia da Universidade do Estado de Mato Grosso (UNEMAT), Rodovia MT 358, Km 7, 78300-000, Tangará da Serra - MT. E-mail: <miriamhinoue@ hotmail.com>;

${ }^{3}$ Eng $^{\mathbf{o}}$ Agr $^{\circ}$, Mestrando em Agronomia (Produção Vegetal), Universidade Federal de Viçosa (UFV), Rodovia MG 230, Km 7, 38810-000, Rio Paranaíba - MG. E-mail: <kassio_mendes_06@hotmail.com>;

${ }^{4}$ Eng $^{\circ}$ Agro, Mestrando em Agronomia (Agricultura), Universidade Estadual Paulista "Júlio de Mesquita Filho" (UNESP). Fazenda Experimental Lageado, 18603-970, Botucatu - SP. E-mail: <clevertontiago@hotmail.com>;

5 Engo Agro, M.Sc., Professora do Departamento de Agronomia da Universidade do Estado de Mato Grosso (UNEMAT), Rodovia MT 358, Km 7, 78300-000, Tangará da Serra $\quad$ MT. E-mail: <anacassiapossamai@hotmail.com>;
} 
and $\left.3.36 \mathrm{~kg} \mathrm{ha}^{-1}\right)$, oxyfluorfen $\left(0 ; 0.48\right.$ and $\left.0.72 \mathrm{~kg} \mathrm{ha}^{-1}\right)$, prometryne $\left(0 ; 0.75\right.$ and $\left.1.50 \mathrm{~kg} \mathrm{ha}^{-1}\right)$ and S-metolachlor $\left(0 ; 1.20\right.$ and $\left.1.44 \mathrm{~kg} \mathrm{ha}^{-1}\right)$ applications. The treatments monitoring was made through sowing of bio-indicator (Brachiaria decumbens or Cucumis sativus). Control was assessed by scores from 0 to $100 \%$ at $0,25,50,75,100$ days after application (DAA). Regardeless of rate, alachlor provided control around $80 \%$ to 66 DAA, in clay soil and up to 50 DAA in sandy. Oxyfluorfen showed control around 80\% to 56 DAA to clay soil and up to 36 DAA to sandy, in both rates. Both rates of prometryne in clay soil provided control over $80 \%$ to 28 DAA, with residual activity higher in recommended rate $\left(1.50 \mathrm{~kg} \mathrm{ha}^{-1}\right)$. In samples sandy, Smetolachlor provided the same control for both rates, controlling the bio-indicator at $80 \%$ up to $52 \mathrm{DAA}$, and for clay soil in rate at $1.44 \mathrm{~kg} \mathrm{ha}^{-1}$, presented $96 \%$ control the 80 DAA. Thereby, there was no increase in residual activity in sandy soil, even with the application of the recommended rate for clay soil.

Key-words: Bio-indicators, persistence, soil texture.

\section{Introdução}

A persistência dos herbicidas no solo varia com a adsorção, lixiviação e degradação e/ou transformação biológica, pois esses fatores regulam a concentração, o fluxo e o tempo de permanência destas moléculas na solução do solo. Tais compostos exercem influência marcante sobre o controle das plantas daninhas, além de causar possíveis injúrias para as culturas em sucessão e o risco de contaminação ambiental (Loux et al., 1989). Contudo, a importância e a intensidade da ação destes fatores sobre a persistência dependem das condições edafoclimáticas específicas aos locais de estudo (Walker et al., 1992).

De modo particular, a persistência dos herbicidas no solo pode ser determinada por métodos biológicos, utilizando como indicador uma planta sensível a um determinado herbicida (bioindicador). Com este método é possível avaliar o tempo do resíduo com bioatividade, podendo assim, além de avaliar seu impacto ambiental, estimar o tempo em que pode permanecer no solo e afetar culturas sensíveis, em um sistema de sucessão ou rotação de culturas (Blanco et al., 2010).

O alachlor é um herbicida não-iônico pertencente ao grupo químico dos cloroacetamidas, registrado no Brasil para controle, em pré-emergência, de plantas daninhas dicotiledôneas e gramíneas em culturas como milho, soja e algodão. Absorção do alachlor ocorre principalmente pelo caulículo das plântulas (Rodrigues \& Almeida, 2005) e possui meia-vida estimada em 15 dias (Flury, 1996).

O herbicida oxyfluorfen pertencente ao grupo químico difeniléter, pouco solúvel em água e fortemente adsorvido nas partículas do solo, por isso é dificilmente lixiviado, permanecendo na camada superficial, onde se decompõe sem deixar resíduos, além de apresentar eficiente controle de várias espécies de plantas daninhas (Pereira, 1987). Yen et al. (2003) estudaram a dissipação e mobilidade do oxyfluorfen em diferentes solos sob diferentes condições de umidade e temperatura e concluíram que altas temperaturas aumentaram a taxa de dissipação.

Prometryne, do grupo químico das triazinas é um herbicida de dissociação básica, para o qual a retenção das moléculas é influenciada principalmente pelo teor de carbono e argila do solo. Apresenta coeficiente de sorção (Koc) entre 400 e $500 \mathrm{~mL} \mathrm{~g}^{-1}$ de solo, fator que o torna sensível as propriedades do solo (Oliveira Jr., 2007).

$\mathrm{O}$ herbicida S-metolachlor é um composto não-ionizável, pertencente ao grupo químico das acetamidas. Aplicado em préemergência ou pré-plantio incorporado para controle de algumas monocotiledôneas e dicotiledôneas, atuando como inibidor da parte 
aérea das plantas, de forma seletiva para as culturas do milho e soja (Ahrens, 1994; Vidal e Fleck, 2001).

Estudos realizados em laboratório mostraram que há grande variabilidade no tempo de permanência do S-metolachlor no solo, sendo que sua meia-vida variou entre 6 e 100 dias, dependendo das condições em que a pesquisa foi realizada (O'Connell et al., 1998; Dinelli et al., 2000; Seybold et al., 2001; Mersie et al., 2004; Accinelli et al., 2005; Ma et al., 2006). Sob condições de campo, trabalhos evidenciaram que a meia-vida do $\mathrm{S}$ metolachlor varia entre 8 e 85 dias (Burgard et al., 1993; O'Connell et al., 1998; Dinelli et al., 2000; Laabs et al., 2002).

A permanência e degradação dos herbicidas no solo são processos-chave na determinação do seu efeito residual (Hinz, 2001). Diante da grande necessidade de conhecimentos sobre a ação dos herbicidas em

Tabela 1. Características físico-químicas das amostras de solos utilizadas nos experimentos. Tangará da Serra e Campo Novo do Parecis, 2011.

\begin{tabular}{|c|c|c|c|c|c|c|c|}
\hline \multirow{2}{*}{ Solo } & \multicolumn{2}{|c|}{$\mathrm{pH}$} & $\mathrm{Al}^{3+}$ & \multirow[t]{2}{*}{$\mathrm{H}^{+}+\mathrm{Al}^{3+}$} & $\mathrm{Ca}^{2+}+\mathrm{Mg}^{2+}$ & \multirow[t]{2}{*}{$\mathrm{Ca}^{2+}$} & \multirow[t]{2}{*}{$\mathrm{K}^{+}$} \\
\hline & $\left(\mathrm{CaCl}_{2}\right)$ & $\left(\mathrm{H}_{2} \mathrm{O}\right)$ & \multicolumn{2}{|c|}{$\left(\mathrm{cmol}_{\mathrm{c}} \mathrm{dm}^{-3}\right)$} & & & \\
\hline $\mathrm{LV}^{\underline{1}}$ & 5,8 & 6,7 & 0 & 3,13 & 6,00 & 4,31 & 0,37 \\
\hline \multirow[t]{3}{*}{$\mathrm{RQ}^{\underline{2}}$} & 7,1 & 7,8 & 0 & 1,13 & 2,75 & 2,19 & 0,02 \\
\hline & \multirow{2}{*}{$\begin{array}{c}\mathrm{P} \\
\left(\mathrm{mg} \mathrm{dm}^{-3}\right)\end{array}$} & \multirow{2}{*}{$\begin{array}{c}\mathrm{C} \\
\left(\mathrm{g} \mathrm{dm}^{-3}\right)\end{array}$} & \multirow{2}{*}{$\begin{array}{c}\mathrm{CTC} \\
\left(\mathrm{cmol}_{\mathrm{c}} \mathrm{dm}^{-3}\right)\end{array}$} & \multirow{2}{*}{$\begin{array}{l}\mathrm{V} \\
(\%)\end{array}$} & Areia & Silte & Argila \\
\hline & & & & & \multicolumn{3}{|c|}{$\left(\mathrm{g} \mathrm{kg}^{-1}\right)$} \\
\hline $\mathrm{LV}^{1 /}$ & 2,3 & 43 & 9,00 & 67,1 & 415 & 147 & 438 \\
\hline $\mathrm{RQ}^{2 /}$ & 46,8 & 15 & 3,90 & 71,0 & 908 & 32 & 60 \\
\hline
\end{tabular}

${ }^{1}$ LV = LATOSSOLO VERMELHO (textura argilosa); ${ }^{2 /} \mathrm{RQ}=$ NEOSSOLO QUARTZARÊNICO (textura arenosa). Fonte: Laboratório de Solos Plante Certo, Várzea Grande, MT.

Cada experimento foi composto por um solo (LATOSSOLO VERMELHO ou NEOSSOLO QUARTZARÊNICO) e um herbicida (alachlor, oxyfluorfen, prometryne e $\mathrm{S}$-metolachlor) em esquema fatorial $3 \times 5$, com quatro repetições, dispostos em blocos casualizados. Doses de herbicidas e época de aplicação foram os fatores estudados. diferentes solos, o objetivo do presente trabalho foi estudar a atividade residual de diferentes doses dos herbicidas alachlor, oxyfluorfen, prometryne e S-metolachlor em solos de textura contrastantes, aplicados em pré-emergência.

\section{Material e métodos}

Oito experimentos foram conduzidos utilizando amostras de solos provenientes de Tangará da Serra (LATOSSOLO VERMELHO - LV, textura argilosa) e Campo Novo do Parecis (NEOSSOLO QUARTZARÊNICO $R Q$, textura arenosa) (Tabela 1), com o intuito de monitorar a atividade residual dos herbicidas alachlor, oxyfluorfen, prometryne e S-metolachlor, em solos com composição e texturas contrastantes. 
ocorreu num período de 0, 25, 50, 75 e 100 dias após a aplicação (DAA) dos herbicidas.

As amostras coletadas em profundidade de 5 a $10 \mathrm{~cm}$ da superfície do solo foram peneiradas, secas ao ar e acondicionadas em vasos plásticos com capacidade para $5 \mathrm{dm}^{3}$. Os herbicidas alachlor $\left(0 ; 2,40\right.$; e $\left.3,36 \mathrm{~kg} \mathrm{ha}^{-1}\right)$, oxyfluorfen $\left(0 ; 0,48 ;\right.$ e $\left.0,72 \quad \mathrm{~kg} \mathrm{ha}^{-1}\right)$, prometryne $\left(0 ; 0,75 ;\right.$ e $\left.1,50 \mathrm{~kg} \mathrm{ha}^{-1}\right)$ e $\mathrm{S}$ metolachlor $\left(0 ; 1,20 ;\right.$ e $\left.1,44 \mathrm{~kg} \mathrm{ha}^{-1}\right)$ foram aplicados com auxílio de um pulverizador costal pressurizado por $\mathrm{CO}_{2}$, munido de bicos tipo leque XR110.02, pressão de trabalho de 2 $\mathrm{kgf} \mathrm{cm}^{-2}$, proporcionando volume de calda equivalente a $200 \mathrm{~L} \mathrm{ha}^{-1}$, de tal forma que a barra do pulverizador estava posicionada a 0,5 $\mathrm{m}$ acima da borda superior dos vasos. Todas as aplicações foram realizadas com temperaturas inferiores à $35^{\circ} \mathrm{C}$ e umidade relativa superior a $60 \%$, com solo úmido.

Após a aplicação dos herbicidas, 10 sementes do bioindicador selecionado (Brachiaria decumbens e Cucumis sativus) foram semeadas aos 0, 25, 50, 75 e 100 DAA, à profundidade de $1 \mathrm{~cm}$. A seleção dessas espécies foi de acordo com a sensibilidade a cada herbicida, determinada em experimentos preliminares (dados não publicados).

Nos períodos entre a aplicação dos herbicidas e a semeadura do bioindicador, foram simuladas precipitações regulares equivalentes à média histórica de 10 anos de freqüência e volume para a região de Tangará da Serra (Martins et al., 2010). Irrigações diárias foram realizadas nos vasos, de 0 até 45 dias após a semeadura (DAS). Também aos 45 DAS, foram feitas avaliações visuais de controle atribuindo notas de 0 a $100 \%$, sendo que, $0 \%$ corresponde a nenhum efeito sobre os bioindicadores e $100 \%$, total controle em relação à testemunha (dose de $0 \quad \mathrm{~kg} \mathrm{ha}^{-1}$ ) (SBCPD, 1995).

Os dados foram submetidos à análise de variância pelo teste $\mathrm{F}$ e ajuste de regressão.
Para análise destes dados foi utilizado o programa Sistema para Análise de Variância (Ferreira, 2003). Para interpretação dos resultados, os valores de controle do bioindicador foram comparados aos do tratamento sem herbicida (dose zero), contudo, quando não houve interação do controle, trabalhou-se com as médias dos tratamentos nas curvas.

\section{Resultados e discussão}

Alachlor

Os resultados de controle de $C$. sativus com a aplicação de alachlor $\left(2,40\right.$ e $3,36 \mathrm{~kg}^{-}{ }^{-}$ 1) nas amostras de LATOSSOLO VERMELHO e NEOSSOLO QUARTZARÊNICO são apresentados na Figura 1.

O herbicida alachlor aplicado diretamente no solo de textura argilosa $(43,8 \%$ de argila) proporcionou controle acima de $80 \%$ até aos 66 DAA, em ambas as doses (Figura 1), controle esse, considerado bom, segundo a escala proposta pela Asociación Latinoamericana de Malezas (1974). Esses dados sugerem a possibilidade de redução nas doses de alachlor, sem afetar o residual.

Em solo de textura argilosa $(>35 \%$ de argila e $18,8 \mathrm{~g} \mathrm{dm}^{-3}$ de carbono orgânico), quando se aplicou a dose recomendada da mistura de alachlor+atrazine $\left(1,43+1,43 \mathrm{~kg}^{-} \mathrm{ha}^{-}\right.$ $\left.{ }^{1}\right)$ e o dobro da dose $\left(2,86+2,86 \mathrm{~kg} \mathrm{ha}^{-1}\right)$, no cultivo de milho híbrido AG-4011, observou-se aos 15 DAA, a inibição de $80 \%$ da biomassa seca de Brassica napus var oleifera (canola) plantada posteriormente a cultura, e a partir dos 90 DAA não houve mais efeito negativo na inibição do acúmulo de biomassa seca (Oliveira Jr., 2001). 


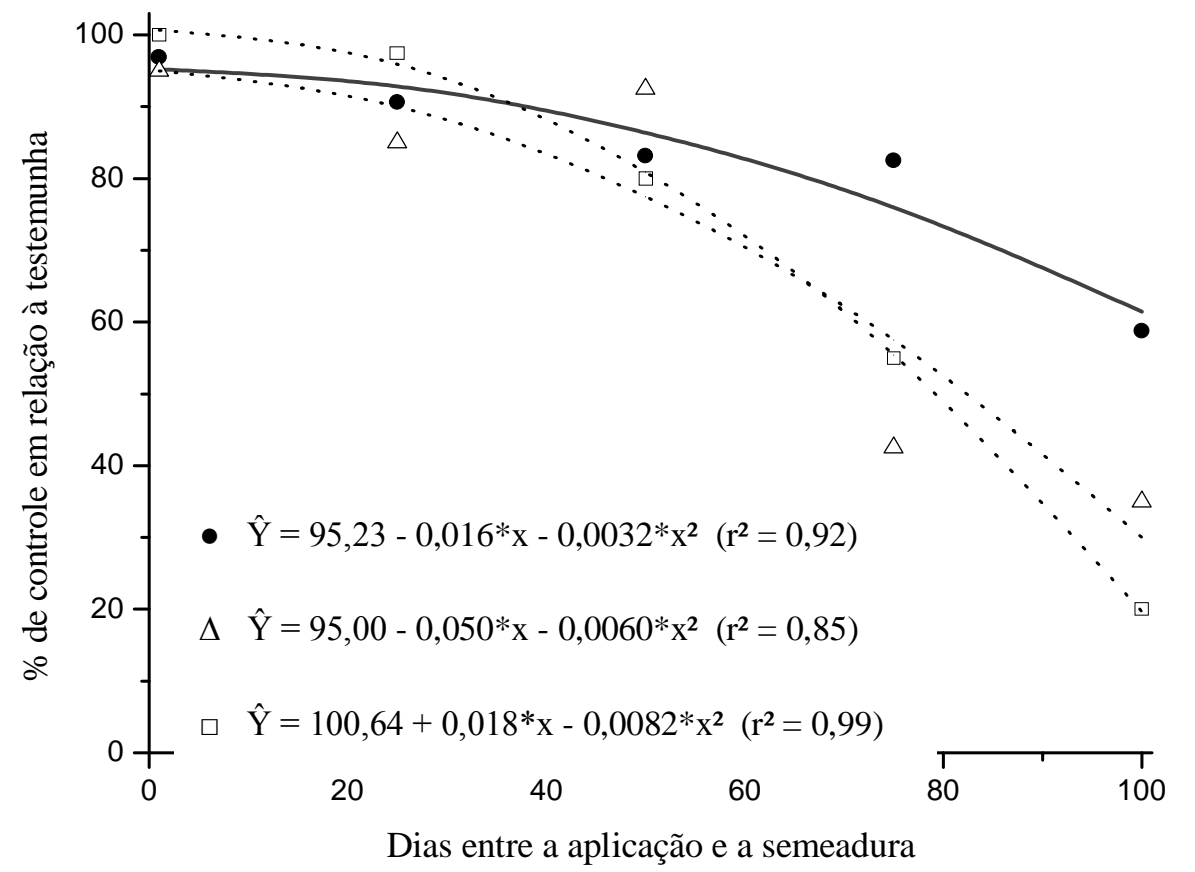

Figura 1. Porcentagem de controle em plantas de C. sativus, em relação à testemunha, em função da aplicação de alachlor aos 0, 25, 50, 75 e 100 dias antes da semeadura. Nos símbolos vazios e linhas tracejadas, os triângulos equivalem aos dados observados para a dose de $3,36 \mathrm{~kg} \mathrm{ha}^{-1} \mathrm{e}$ os quadrados para a dose de $2,40 \mathrm{~kg} \mathrm{ha}^{-1}$ no NEOSSOLO QUARTZARÊNICO (textura arenosa). Os círculos sólidos e linhas cheias equivalem à média dos dados observados para ambas as doses $\left(2,40\right.$ e $\left.3,36 \mathrm{~kg} \mathrm{ha}^{-1}\right)$ no LATOSSOLO VERMELHO (textura argilosa).

Quando utilizada a dose recomendada $\left(1,2 \mathrm{~kg} \mathrm{ha}^{-1}\right)$ de alachlor, Raimondi et al. (2010) verificaram que houve aumento considerável da atividade residual para o controle da espécie Amaranthus viridis, para a qual se obteve controle efetivo $(\geq 80 \%)$ até aos 30 DAA, em solo de textura francoargiloarenosa (20\% de argila).

Em amostras de NEOSSOLO QUARTZARÊNICO o comportamento das duas doses também foi semelhante, havendo controle acima de $80 \%$ em torno de 50 DAA (Figura 1). Raimondi et al. (2010) ao compararem a dose $\left(0,596 \mathrm{~kg} \mathrm{ha}^{-1}\right)$ com a dose recomendada $\left(1,2 \mathrm{~kg} \mathrm{ha}^{-1}\right)$ para Amaranthus spinosus, observaram que, mesmo mediante aumento de $102 \%$ da dose, não houve aumento no período de atividade residual.

Alachlor aplicado em solo com baixos teores de argila resultou em menor atividade residual, mesmo com emprego de doses elevadas, provavelmente devido a perda por lixiviação. Dados de fitointoxicação de $C$. sativus indicaram que as moléculas de alachlor $\left(2,40 \mathrm{~kg} \mathrm{ha}^{-1}\right)$ atingiram a camada de $10-15 \mathrm{~cm}$ de profundidade nas lâminas de 80 e $100 \mathrm{~mm}$, em NEOSSOLO QUARTZARÊNICO $(90,8 \%$ de areia), ressaltando a mobilidade deste herbicida em solo com textura arenosa (Inoue et al., 2010). Conforme constatado por Bowman (1990), o alachlor também apresentou 
mobilidade quando aplicado em lisímetro $0,72 \mathrm{~kg} \mathrm{ha}^{-1}$ ) aplicado em LATOSSOLO composto de areia.

\section{Oxyfluorfen}

Os resultados de controle de $B$. VERMELHO N NEOSSOLO QUARTZARÊNICO são apresentados na Figura 2.

decumbens com o uso de oxyfluorfen $(0,48$ e

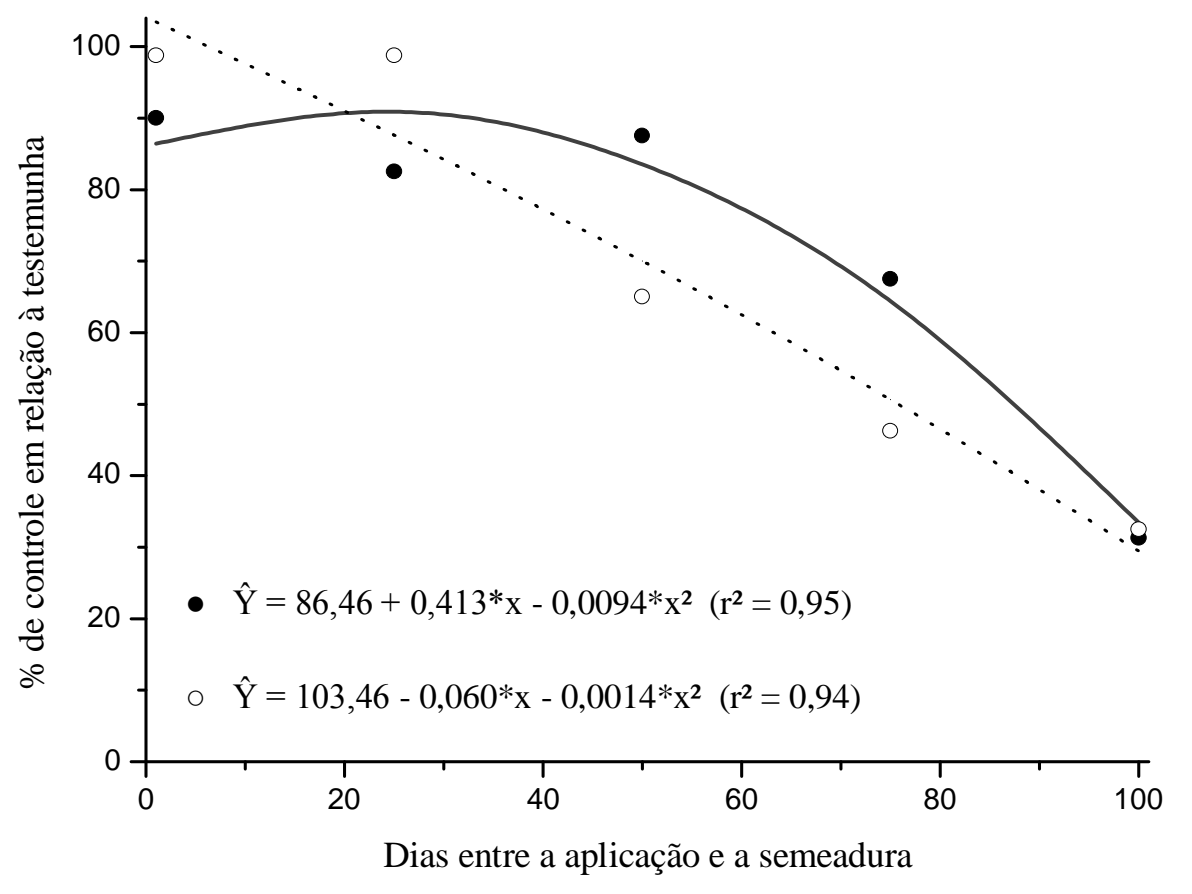

Figura 2. Porcentagem de controle em plantas de B. decumbens, em relação à testemunha, em função da aplicação de oxyfluorfen aos $0,25,50,75$ e 100 dias antes da semeadura. Os círculos equivalem à média dos dados observados para ambas as doses $\left(0,48 \mathrm{e} 0,72 \mathrm{~kg} \mathrm{ha}^{-1}\right)$. Símbolos sólidos e linhas cheias equivalem à média dos dados no LATOSSOLO VERMELHO (textura argilosa) e os símbolos vazios e linhas tracejadas no NEOSSOLO QUARTZARÊNICO (textura arenosa).

Verificou-se que, independente da dose avaliada, o herbicida oxyfluorfen proporcionou o mesmo comportamento, sendo que houve controle acima de $80 \%$ até aos 56 DAA para amostras de LATOSSOLO VERMELHO (43,8\% de argila e $43 \mathrm{~g} \mathrm{dm}^{-3}$ de MO) e até aos 36 DAA para NEOSSOLO QUARTZARÊNICO (6\% de argila e $15 \mathrm{~g} \mathrm{dm}^{-3}$ de MO), diminuindo gradativamente após esse período (Figura 2).
Costa et al. (2002) verificaram que houve controle eficiente de $B$. decumbens até 62 DAT (dias após aplicação dos tratamentos), com a aplicação (pré-emergência) de oxyfluorfen nas doses 0,48 e $0,24 \mathrm{~kg} \mathrm{ha}^{-1}$ em LATOSSOLO VERMELHO-ESCURO $(67 \%$ de argila).

Silva et al. (2002) observaram aos 30 DAA, controle de $93 \%$ e $95 \%$ de $B$. decumbens com a aplicação de oxyfluorfen, 
respectivamente nas doses de 0,48 e $0,96 \mathrm{~kg} \mathrm{ha}^{-}$ 1 em Podzólico Vermelho-Amarelo Câmbico, constituído por $66 \%$ de areia, $13 \%$ de silte, $34 \%$ de argila e $2,11 \%$ MO.

Assim, considerando os dois tipos de solos, os resultados indicaram que é possível obter controle satisfatório com a utilização de doses menores de oxyfluorfen $\left(0,48 \mathrm{~kg} \mathrm{ha}^{-1}\right)$ para controle de B. decumbens.

Melo et al. (2010), nas avaliações de persistência do herbicida oxyfluorfen $(0,72 \mathrm{~kg}$ $\mathrm{ha}^{-1}$ ), observaram que cerca de $50 \%$ de controle em plantas de Sorghum bicolor aos 60 DAA, em solo de textura argilosa $\left(90 \mathrm{~g} \mathrm{dm}^{-3}\right.$ de MO e $56 \%$ de argila), e $90 \%$ de controle aos 60 DAA em solo de textura franco-arenosa $\left(4 \mathrm{~g} \mathrm{dm}^{-3} \mathrm{de}\right.$ MO e $14 \%$ de argila). Em contrariedade, independentemente da dose $\left(0,48\right.$ e $0,72 \mathrm{~kg} \mathrm{ha}^{-}$ ${ }^{1}$ ), a atividade residual de oxyfluorfen variou conforme a textura e o teor de matéria orgânica das amostras de solo.

\section{Prometryne}

A Figura 3 apresenta os dados de controle de $C$. sativus após a aplicação de 0,75 e $1,50 \mathrm{~kg} \mathrm{ha}^{-1}$ de prometryne nas amostras de LATOSSOLO VERMELHO e NEOSSOLO QUARTZARÊNICO.

A maior dose de prometryne $(1,50 \mathrm{~kg}$ $\mathrm{ha}^{-1}$ ) aplicadas em solo de textura argilosa (43 $\mathrm{g} \mathrm{dm}^{-3} \mathrm{MO}$ e $43,8 \%$ de argila) proporcionou controle eficiente de $90 \%$ até aos 37 DAA (Figura 3), reduzindo o efeito do herbicida no solo argiloso à medida que se aumentou o período após a aplicação do herbicida prometryne. Aos 60 DAA, o prometryne atingiu apenas $60 \%$ de controle na menor dose $\left(0,75 \mathrm{~kg} \mathrm{ha}^{-1}\right)$, contudo, a dose recomendada $\left(1,50 \mathrm{~kg} \mathrm{ha}^{-1}\right)$ para solo argiloso proporcionou controle acima de $80 \%$ no mesmo período (Figura 3).

Em solo de textura arenosa, independentemente da dose $\left(0,75\right.$ e $1,50 \mathrm{~kg} \mathrm{ha}^{-}$ ${ }^{1}$ ), prometryne, proporcionou controle de $70 \%$ do bioindicador até aos 57 DAA (Figura 3).

Segundo Raimondi et al. (2010), a utilização da dose recomendada de prometryne $\left(0,9 \mathrm{~kg} \mathrm{ha}^{-1}\right)$ prolongou a atividade residual até aos 29 DAA e em dose menor $\left(0,601 \mathrm{~kg} \mathrm{ha}^{-1}\right)$ até aos 20 DAA, proporcionando controle mínimo de $80 \%$ para Amaranthus hybridus, em solo de textura franco-argiloarenosa $(20 \%$ de argila).

Portanto, esses resultados demonstram que a atividade residual de prometryne é extremamente dependente da dose utilizada, devendo-se evitar o emprego de subdosagens (Raimondi et al., 2010).

Estudos de Prata et al. (2001) evidenciaram que herbicidas do grupo das triazinas, como o prometryne, apresentam de baixa a média solubilidade em água e reduzida velocidade de degradação, fatores que podem aumentar a persistência da molécula no solo e a probabilidade de contaminação de lençóis freáticos. 


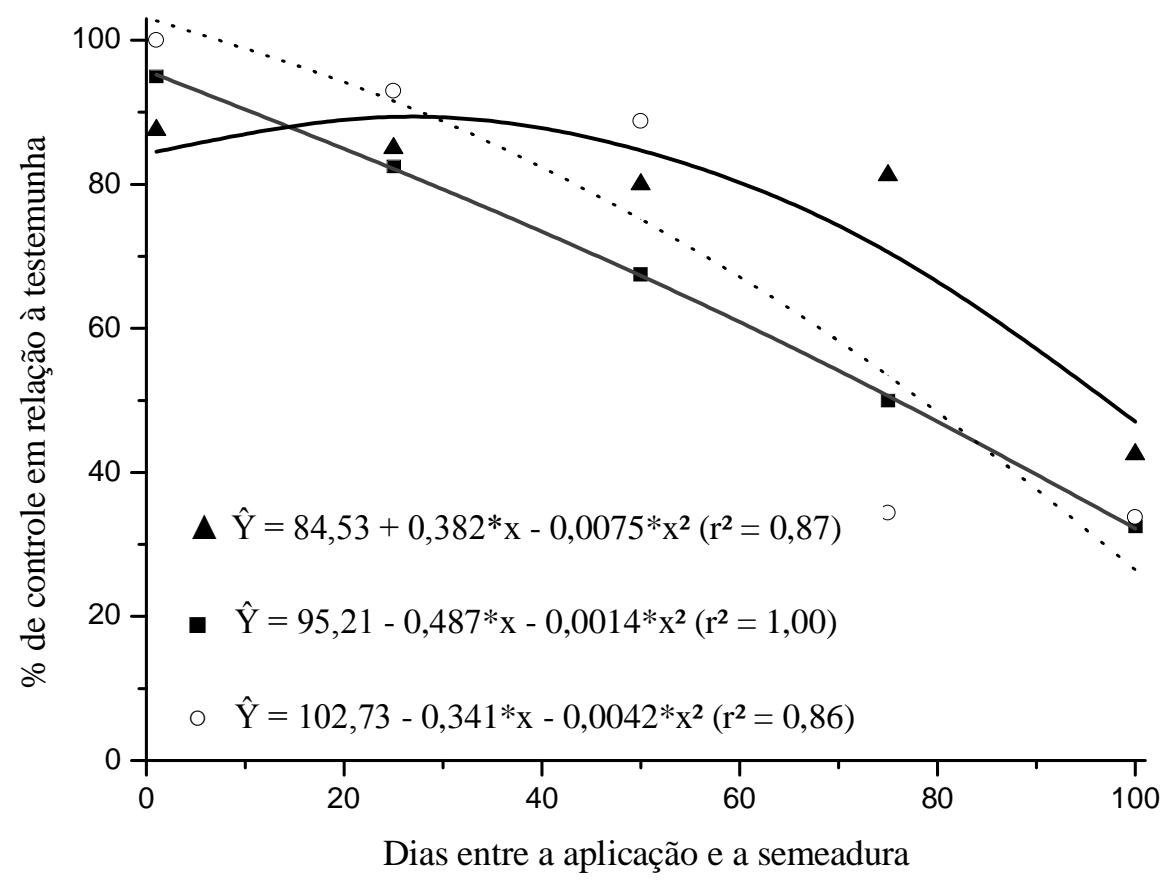

Figura 3. Porcentagem de controle em plantas de $C$. sativus, em relação à testemunha, em função da aplicação de prometryne aos 0, 25, 50, 75 e 100 dias antes da semeadura. Os círculos vazios e linhas tracejadas equivalem à média dos dados observados para ambas as doses $(0,75 \mathrm{e} 1,50 \mathrm{~kg}$ $\mathrm{ha}^{-1}$ ) no NEOSSOLO QUARTZARÊNICO (textura arenosa). Nos símbolos sólidos e linhas cheias, os triângulos equivalem aos dados observados para a dose de $1,50 \mathrm{~kg} \mathrm{ha}^{-1}$ e os quadrados para a dose de $0,75 \mathrm{~kg} \mathrm{ha}^{-1}$ no LATOSSOLO VERMELHO (textura argilosa).

\section{S-metolachlor}

A Figura 4 indica os resultados de controle de B. decumbens obtidos após o uso do herbicida S-metolachlor $\left(1,20\right.$ e 1,44 $\mathrm{kg}^{-}$ha $^{-}$ 1) em LATOSSOLO VERMELHO e NEOSSOLO QUARTZARÊNICO.

Nas amostras de textura arenosa, este herbicida proporcionou o mesmo controle para ambas as doses, controlando o bioindicador em $80 \%$ até 52 DAA. Para o solo de textura argilosa a persistência do S-metolachlor foi elevada para a dose $1,44 \mathrm{~kg} \mathrm{ha}^{-1}$, com $96 \%$ de controle aos 80 DAA (Figura 4).

O S-metolachlor aplicado no solo argiloso ( $\mathrm{MO} 43 \mathrm{~g} \mathrm{dm}^{-3}$ ) proporcionou bom controle até aos 74 DAA, mesmo na dose de
$1,20 \mathrm{~kg} \mathrm{ha}^{-1}$. Na dose recomendada $\left(1,44 \mathrm{~kg} \mathrm{ha}^{-}\right.$ $\left.{ }^{1}\right)$ o herbicida proporcionou controle acima de $90 \%$ até aos 100 DAA (Figura 4). Esses valores estão relacionados com o baixo potencial de lixiviação do S-metolachlor que geralmente é insignificante quando o conteúdo de matéria orgânica do solo é maior que $20 \mathrm{~g}$ $\mathrm{dm}^{-3}$ (Ahrens, 1994), mesmo não apresentando valor elevado de Koc (200 $\mathrm{mL} \mathrm{g}^{-1}$ de solo) e mostrando solubilidade em água considerada elevada (448 $\mathrm{mL} \mathrm{L}^{-1}$ ) (Vieira e Silva, 1998; Rodrigues e Almeida, 2005).

Freitas et al. (2006a), em um solo argiloso (MO 27,9 $\mathrm{g} \mathrm{dm}^{-3}$ ), avaliando doses e combinações de herbicidas em pré e pósemergência, concluíram que a aplicação em 
pré-emergência de S-metolachlor na dose de $1,152 \mathrm{~kg} \mathrm{ha}^{-1}$ foi eficiente no controle das plantas daninhas Alternanthera tenella, Bidens spp., Acanthospermum hispidum e Ipomea grandifolia, permitindo a colheita do algodão com menor interferência das plantas daninhas.

Corroborando com esses dados, Freitas et al. (2006b), observaram que ao aplicarem $1,152 \mathrm{~kg} \mathrm{ha}^{-1}$ de S-metolachlor em préemergência, 7,875 $\mathrm{g} \mathrm{ha}^{-1}$ de trifloxysufuronsodium aos 18 dias após a emergência do algodão (DAE), obtem-se nível de controle superior a $90 \%$ para Alternanthera tenella até 60 DAE em solo de textura argilosa com 27,9 g $\mathrm{dm}^{-3}$ de matéria orgânica, evidenciando novamente a possibilidade de utilização de menores doses, mantendo controle eficiente.

Em solo de textura argilosa (43,8\% de argila), o herbicida S-metolachlor tendeu a persistir por um período maior, quando comparado com solo de textura arenosa (6\% de argila), mostrando que a quantidade de argila tem influenciado no comportamento do herbicida no solo.

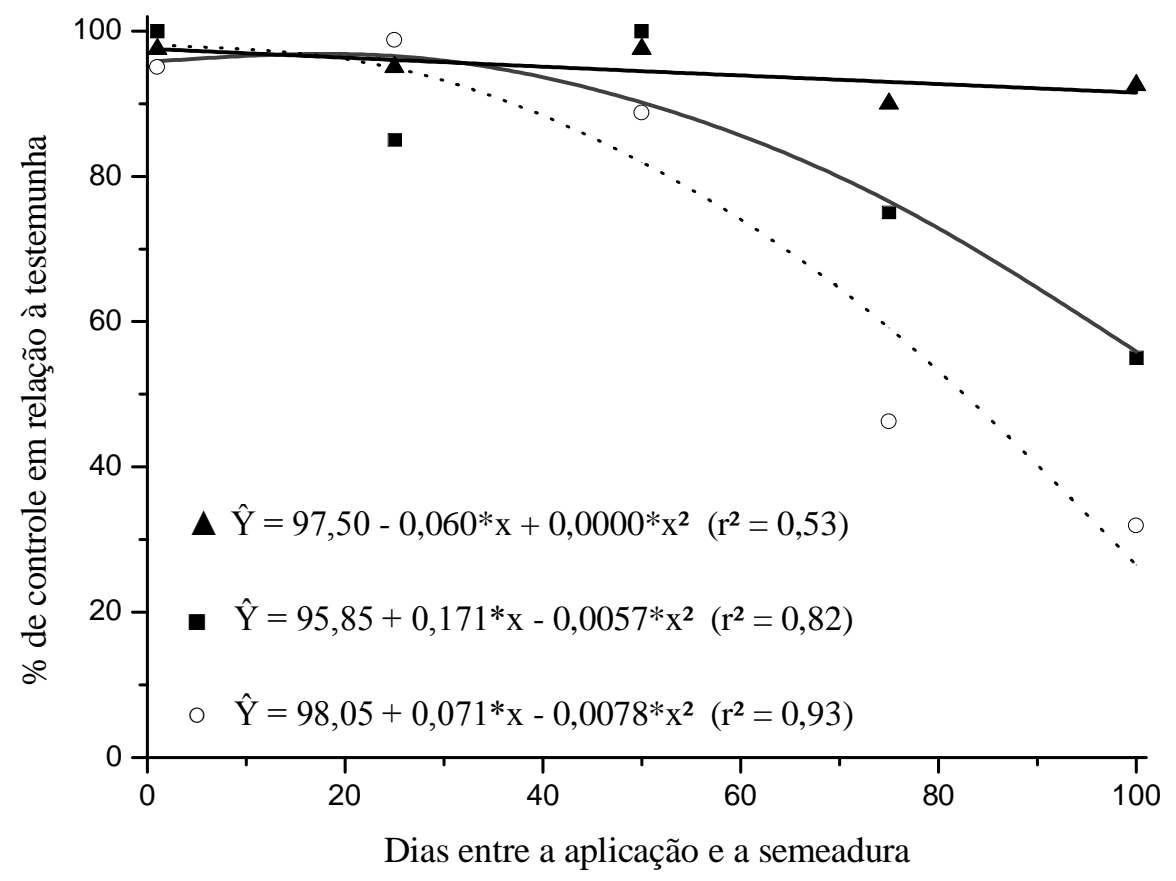

Figura 4. Porcentagem de controle em plantas de $B$. decumbens, em relação à testemunha, em função da aplicação de S-metolachlor aos 0, 25, 50, 75 e 100 dias antes da semeadura. Os círculos vazios e linhas tracejadas equivalem à média dos dados observados para ambas as doses $\left(1,20\right.$ e $\left.1,44 \mathrm{~kg} \mathrm{ha}^{-1}\right)$ no NEOSSOLO QUARTZARÊNICO (textura arenosa). Nos símbolos sólidos e linhas cheias, os triângulos equivalem aos dados observados para a dose de $1,44 \mathrm{~kg} \mathrm{ha}^{-1}$ e os quadrados para a dose de 1,20 $\mathrm{kg} \mathrm{ha}^{-1}$ no LATOSSOLO VERMELHO (textura argilosa).

\section{Conclusão}

O incremento da dose de alachlor não aumentou a atividade residual do produto em LATOSSOLO VERMELHO e foi semelhante em NEOSSOLO QUARTZARÊNICO. Independente da dose $\left(0,48\right.$ e $\left.0,72 \mathrm{~kg} \mathrm{ha}^{-1}\right)$, a atividade residual de oxyfluorfen foi prolongada conforme o aumento do teor de 
argila e matéria orgânica presente no solo. Em solo argiloso, a atividade residual de prometryne foi maior quando utilizado a dose recomendada $\left(1,50 \mathrm{~kg} \mathrm{ha}^{-1}\right)$. O aumento da dose de S-metolachlor não prolonga a atividade residual em solo de textura arenosa.

\section{Agradecimentos}

Os autores agradecem à FAPEMAT e ao CNPq pelo auxílio financeiro dado a essa pesquisa.

\section{Referências}

ACCINELLI, C.; SCREPANTI, C.; VIARI, A. Influence of flooding on the degradation of linuron, isoproturon and metolachlor in soil. Agronomy for Sustainable Development, v.25, n.3, p.401-406, 2005.

AHRENS, W.H. Herbicide handbook. 7. ed. Lawrence: Weed Science Society of America, 1994, 352p.

ALAM, Asociacion Latinoamericana De Malezas. Recomendaciones sobre unificación de evaluación en ensayos de control de malezas. ALAM, v.1, n.1, p.35-8, 1974.

BLANCO, F.M.G.; VELINI, E.D.; FILHO, A.B. Persistência do herbicida sulfentrazone em solo cultivado com cana-de-açúcar. Bragantia, v.69, n.1, p.71-75, 2010.

BOWMAN, B.T. Mobility and persistence of alachlor, atrazine and metolachlor in plainfield sand, and atrazine and isazofos in honeywood silt loam, using field lysimeters. Environmental Toxicology \& Chemistry, v. 9, p. 453-461, 1990.

BURGARD, D.J. et al. Metolachlor distribution in a sandy soil under irrigated potato production. Weed Science, v.41, n.4, p.648-655, 1993.

COSTA, E.A.D. et al. Eficiência de nova formulação do herbicida oxyfluorfen no controle de plantas daninhas em área de Pinus caribea Morelet var. hondurensis Barr. et Golf. Revista Árvore, v.26, n.6, p.683-689, 2002.

DINELLI, G. et al. Comparison of the persistence of atrazine and metolachlor under field and laboratory conditions. Journal of Agricultural and Food Chemistry, v.48, n.7, p.3037-3043, 2000.

FERREIRA, D.F. Programa Sisvar.exe: Sistema de Análise de Variância. Versão 3.04. Lavras: UFLA, 2003.

FLURY, M. Experimental evidence of transport of pesticides through field soils - a review. Journal of Environmental Quality, v.25, n. 1, p.25-45, 1996.

FREITAS, R.S. et al. Manejo de plantas daninhas na cultura do algodoeiro com Smetolachlor e trifloxysulfuron-sodium em sistema de plantio convencional. Planta Daninha, v.24, n.2, p.311-318, $2006 a$.

FREITAS, R.S. et al. Manejo de plantas daninhas na cultura do algodoeiro em sistema de plantio direto. Planta Daninha, v.24, n.2, p.339-346, $2006 \mathrm{~b}$.

HINZ, C. Description of sorption data with isotherm equations. Geoderma, v.99, n.3/4, p.225-243, 2001.

INOUE, M.H et al. Potencial de lixiviação de herbicidas utilizados na cultura do algodão em colunas de solo. Planta Daninha, v.28, n.4, p.825-833, 2010.

LAABS, V. et al. Fate of pesticides in tropical soils of Brazil under field conditions. Journal of Environmental Quality, v.31, n.1, p.256268, 2002.

LOUX, M.M.; LIEBL, R.A.; SLIFE, F.W. Availability and persistence of imazaquin, imazethapyr, and clomazone in soil. Weed Science, v.37, n.1, p.259-267, 1989.

MA, Y.; LIU, W.P.; WEN, Y.Z. Enantioselective degradation of rac- 
metolachlor and S-metolachlor in soil. Pedosphere, v.16, n.4, p.489-494, 2006.

MARTINS, J.A. et al. Probabilidade de precipitação para a microrregião de Tangará da Serra, Estado do Mato Grosso. Pesquisa Agropecuária Tropical, v.40, n.3, p.291-296, 2010.

MELO, C.A.D. et al. Efeito residual de sulfentrazone, isoxaflutole e oxyfluorfen em três solos. Planta Daninha, v.28, n.4, p.835842, 2010.

MERSIE, W. et al. Degradation of metolachlor in bare and vegetated soils and in simulated water-sediment systems. Environmental Toxicology \& Chemistry, v.23, n.11, p.26272632, 2004.

O'CONNELL, P.J.; HARMS, C.T.; ALLEN, J.R.F. Metolachlor, S-metolachlor and their role within sustainable weed-management. Crop Protection, v.17, n.3, p.207-212, 1998.

OLIVEIRA JR., R.S. Atividade residual de imazaquin e alachlor+atrazine para plantio seqüencial de canola. Ciência Rural, v.31, n.2, p.219-224, 2001.

OLIVEIRA JR., R.S. Comportamento dos herbicidas residuais no solo: relação entre parâmetros físico-químicos e atributos do solo. Maringá: EDUEM, 2007.

PEREIRA, W.S.P. Herbicida de préemergência oxifluorfen. Série Técnica IPEF, v.4, n.12, p.45-60, 1987.

PRATA, F. et al. Degradação e sorção de ametrina em dois solos com aplicação de vinhaça. Pesquisa Agropecuária Brasileira, v.36, n.7, p.975-981, 2001.

RAIMONDI, M.A. et al. Atividade residual de herbicidas aplicados ao solo em relação ao controle de quatro espécies de Amaranthus. Planta Daninha, v.28, n.spe, p.1073-1085, 2010.
RODRIGUES, B.N.; ALMEIDA, F.S. Guia de herbicidas. 5. ed. Londrina: Autores, 2005, $591 \mathrm{p}$.

SBCPD, Sociedade Brasileira da Ciência das Plantas Daninhas. Procedimentos para instalação, avaliação e análise de experimentos com herbicidas. Londrina: SBCPD, 1995.

SEYBOLD, C.A.; MERSIE, W.; MCNAMEE, C. Anaerobic degradation of atrazine and metolachlor and metabolite formation in wetland soil and water microcosms. Journal of Environmental Quality, v.30, n.4, p.12711277, 2001.

SILVA, W. et al. Redução da interferência de Brachiaria decumbens na formação de pastagem com Penisetum purpureum através de herbicidas. Planta Daninha, v.20, n.2, p.273-281, 2002.

VIDAL, R.A.; FLECK, N.G. Inibidores do crescimento da parte aérea. In: VIDAL, R.A.; MEROTO JR., A. (Orgs.). Herbicidologia. Porto Alegre: Evangraf, 2001. p.123-130.

VIEIRA, R.F.; SILVA, A.A. Aplicação de defensivos via água de irrigação por aspersão. In: VIEIRA, C.; PAULA JR., T.J.; BORÉM, A. Feijão - aspectos gerais e cultura no Estado de Minas. Viçosa: UFV, 1998. p.267-323.

WALKER, A.; MOON, Y.; WELCH, S.J. Influence of temperature, soil moisture, and soil characteristics on the persistence of alachlor. Pesticide Science, v.35, n.2, p.109116, 1992.

YEN, J.H.; SHEU, W.S.; WANG, Y.S. Dissipation of the herbicide oxifluorfen in subtropical soils and its potential to contaminate groundwater. Journal

Ecotoxicology and Environmental, v.54, n.2, p.151-156, 2003. 